\title{
On the strain-energy density in linear elasticity
}

\author{
C. O. HORGAN*
}

Department of Engineering Mechanics, The University of Michigan, Ann Arbor, Michigan, U.S.A.

(Received May 23, 1972)

\section{SUMMARY}

Standard results from matrix theory are used to derive optimal upper and lower bounds for the strain-energy density in terms of the norm of the stress tensor in two and three dimensions. The approach also yields directly necessary and sufficient conditions for positive-definiteness.

\section{Introduction}

In the course of a recent investigation of Saint-Venant's principle in linear anisotropic plane elastostatics [1], the question of finding optimal upper and lower bounds for the strain-energy density in terms of the norm of the stress tensor arose naturally. Similar considerations were treated by Knowles and Sternberg [2], Toupin [3] and Roseman [4]. In the present note we use standard results of matrix analysis to obtain such bounds in two and three dimensions. In addition, this approach yields directly necessary and sufficient conditions for positive--definiteness of the strain energy.

\section{Three-dimensional elasticity}

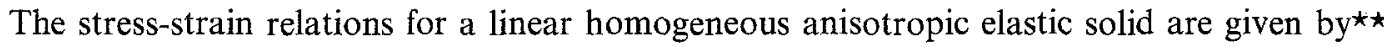

$$
\tau_{i j}=c_{i j k l} e_{k l} \quad(i, j, k, l=1,2,3),
$$

where the components $c_{i j k l}$ of the constant elasticity tensor c satisfy the symmetry relations

$$
c_{i j k l}=c_{j i k l}=c_{k l i j} .
$$

Here $\tau_{i j}=\tau_{j i}$ are the Cartesian components of the stress tensor $\tau$ and $e_{i j}$ the components of the strain tensor defined by $e_{i j}=\frac{1}{2}\left(u_{i, j}+u_{i, i}\right)$, where $u_{i}$ are the components of the displacement vector-field. We assume that the determinant $\left|c_{i j k l}\right| \neq 0$ so that (2.1) has a unique inverse

$$
e_{i j}=s_{i j k l} \tau_{k l},
$$

where the elastic compliances $s_{i j k l}$ also satisfy relations (2.2). The strain-energy density $W$ is given by

$$
2 W=\tau_{i j} e_{i j}=s_{i j k l} \tau_{i j} \tau_{k l}
$$

a quadratic form in the symmetric stress components $\tau_{i j}$. We say that $W(\tau)$ is positive definite if $W(\tau)>0$ for all $\tau \neq 0$.

We introduce the matrix notation $2 W=t^{\prime} S t$, where $t$ is the column vector

* Now at School of Mathematics and Physics, University of East Anglia, Norwich, England.

$\star \star$ See Sokolnikoff [5] for example. Cartesian tensor notation is used throughout, with summation over repeated subscripts implied. 


$$
t=\left[\begin{array}{l}
\tau_{11} \\
\tau_{22} \\
\tau_{33} \\
\tau_{23} \sqrt{ } 2 \\
\tau_{31} \sqrt{ } 2 \\
\tau_{12} \sqrt{ } 2
\end{array}\right]
$$

$t^{\prime}$ is the transpose of $t$ and the $6 \times 6$ symmetric matrix $S=\left[s_{p q}\right],(p, q=1,2, \ldots 6)$ is defined by

$$
\begin{aligned}
s_{p q} & =s_{i j k l} \quad\left(\begin{array}{l}
p, q=1,2,3 \\
i=j, \quad k=l
\end{array}\right), \\
& =2 s_{i j k l} \quad\left(\begin{array}{l}
p, q=4,5,6 \\
i \neq j, \quad k \neq l
\end{array}\right), \\
& =s_{i j k l} \sqrt{ } 2 \quad\left(\begin{array}{l}
p=1,2,3 ; q=4,5,6 \\
i=j, \quad k \neq l
\end{array}\right)
\end{aligned}
$$

The norm of the stress tensor is given by $|\tau|=\left(\tau_{i j} \tau_{i j}\right)^{\frac{1}{2}}=\left(t^{\prime} t\right)^{\frac{1}{2}}$. One of the criteria for positivedefiniteness of $W$ which we use is provided by the following well known result from matrix analysis. (See Bellman [6], for example.)

Lemma 1. The quadratic form $W$ is positive definite if and only if all the eigenvalues $\lambda$ of $S$ are positive.

Furthermore, it can be readily shown from the standard diagonalization of real symmetric matrices [6] that $W$ satisfies

$$
\lambda_{\min }|\tau|^{2} \leqq 2 W \leqq \lambda_{\max }|\tau|^{2}
$$

where $\lambda_{\min }, \lambda_{\max }$ are the minimum and maximum eigenvalues respectively of $S$ and $|\tau|^{2}=\tau_{i j} \tau_{i j}=$ $t^{\prime} t$. These upper and lower bounds are optimal in the sense that $\tau$ may be chosen (as the eigenvector corresponding to $\lambda_{\min }, \lambda_{\max }$ respectively) to yield equality in (2.7). Explicit determination of the eigenvalues $\lambda$ in the fully anisotropic case is difficult in general; however, the results from matrix theory on bounds for eigenvalues (see, for example, Marcus and Minc [7]) may be used in (2.7) to yield upper and lower bounds (no longer optimal) for $W$.

Isotropic case. Here (2.3) becomes (see [5])

$$
e_{i j}=\frac{1}{2 \mu}\left[\tau_{i j}-\frac{\sigma}{1+\sigma} \cdot \delta_{i j} \tau_{k k}\right],
$$

where $\mu$ is the shear modulus, $\sigma$ is Poisson's ratio and $\delta_{i j}$ the Kronecker delta. The matrix $S$ is given by

$$
2 \mu S=\left[\begin{array}{cccccc}
\frac{1}{1+\sigma} & \frac{-\sigma}{1+\sigma} & \frac{-\sigma}{1+\sigma} & 0 & 0 & 0 \\
\frac{-\sigma}{1+\sigma} & \frac{1}{1+\sigma} & \frac{-\sigma}{1+\sigma} & 0 & 0 & 0 \\
\frac{-\sigma}{1+\sigma} & \frac{-\sigma}{1+\sigma} & \frac{1}{1+\sigma} & 0 & 0 & 0 \\
0 & 0 & 0 & 1 & 0 & 0 \\
0 & 0 & 0 & 0 & 1 & 0 \\
0 & 0 & 0 & 0 & 0 & 1
\end{array}\right]
$$


The eigenvalues $\lambda$ are the zeros of

so that

$$
\left(\frac{1}{2 \mu}-\lambda\right)^{3}\left(\frac{1-2 \sigma}{2 \mu(1+\sigma)}-\lambda\right)\left(\frac{1}{2 \mu}-\lambda\right)^{2}
$$

$$
\lambda=\frac{1}{2 \mu}, \frac{1-2 \sigma}{2 \mu(1+\sigma)}
$$

the first occurring with multiplicity five. Application of Lemma 1 immediately yields the result that $W$ is positive definite if and only if

$$
\mu>0, \quad-1<\sigma<\frac{1}{2} \text {. }
$$

The restrictions (2.10) are the familiar inequalities $\star$ invoked in the classical Kirchhoff uniqueness theorem of elastostatics.

The upper and lower bounds (2.7), on using (2.9), lead to the following theorem.

Theorem 1. Let

$$
W(\tau)=\frac{1}{4 \mu}\left(\tau_{i j} \tau_{i j}-\frac{\sigma}{1+\sigma} \tau_{i i} \tau_{j j}\right)
$$

be the strain-energy density associated with a stress tensor $\tau$ and let $W$ be positive definite. Then

$$
\frac{c(\sigma)}{2 \mu}|\tau|^{2} \leqq 2 W \leqq \frac{b(\sigma)}{2 \mu}|\tau|^{2}
$$

where

$$
c(\sigma)=\min \left[1, \frac{1-2 \sigma}{1+\sigma}\right], \quad b(\sigma)=\max \left[1, \frac{1-2 \sigma}{1+\sigma}\right]
$$

or, equivalently,

$$
\begin{aligned}
& c(\sigma)=1, \quad b(\sigma)=\frac{1-2 \sigma}{1+\sigma}(-1<\sigma<0), \\
& c(\sigma)=\frac{1-2 \sigma}{1+\sigma}, b(\sigma)=1 \quad\left(0 \leqq \sigma<\frac{1}{2}\right) .
\end{aligned}
$$

The lower bound in (2.12) was derived in Lemma 1 of [2], while for $0<\sigma<\frac{1}{2}(2.12)$ also appeared in [4]. A similar result was obtained by Toupin in [3] $]^{\star \star}$. The estimate (2.12) is optimal in the sense that we obtain equality on the left if we take $\tau_{i i}=0, \tau_{i j}$ arbitrary when $-1<\sigma<0$ and if we chose $\tau_{i j}=\tau \delta_{i j}$ when $0<\sigma<\frac{1}{2}$. Interchange of these eigenvectors yields equality on the right. Finally, we note that for $\sigma=0$ we get equality on both sides of.(2.12) for any stress tensor $\tau$.

\section{Two-dimensional case}

In the well known theory of plane strain for an isotropic elastic solid [5], the strain-energy density is given by

$$
W=\frac{1}{4 \mu}\left(\tau_{\alpha \beta} \tau_{\alpha \beta-} \sigma \tau_{\alpha \alpha} \tau_{\beta \beta}\right)
$$

where the Greek subscripts range over the integers 1,2 . As before, we write $2 W=t^{\prime} S t$, where now we have

$$
t=\left[\begin{array}{l}
\tau_{11} \\
\tau_{22} \\
\tau_{12} \sqrt{2}
\end{array}\right], \quad 2 \mu S=\left[\begin{array}{lll}
1-\sigma & -\sigma & 0 \\
-\sigma & 1-\sigma & 0 \\
0 & 0 & 1
\end{array}\right] .
$$

* These are usually derived by other methods in elasticity texts.

$\star \star$ Note also the footnote on p. 88 of [3], referring to a remark due to Ericksen. 
The eigenvalues $\lambda$ of $\mathrm{S}$ are easily found to be

$$
\lambda=\frac{1}{2 \mu}, \frac{1-2 \sigma}{2 \mu},
$$

the first occurring with multiplicity two. Lemma 1 now gives the result that $W$ is positive definite if and only if

$$
\mu>0, \quad \sigma<\frac{1}{2} \text {. }
$$

Notice that the restrictions (3.4) are less severe than the analogous (2.10) in the three-dimensional case. This was also noted by Knops and Payne [8] in their treatment of the analog of Kirchhoff's uniqueness theorem for the basic boundary value problems of plane strain. (See $\S 5.1$ of [8].)

Finally, we use (2.7) and (3.3) to obtain the optimal upper and lower bounds furnished by the following theorem.

Theorem 2. Let $W$ be the strain-energy density (3.1) associated with the stress tensor $\tau$ in plane strain and let $W$ be positive definite.

Then

$$
\frac{c(\sigma)}{2 \mu}|\tau|^{2} \leqq 2 W \leqq \frac{b(\sigma)}{2 \mu}|\tau|^{2}, \quad\left(|\tau|^{2}=\tau_{\alpha \beta} \tau_{\alpha \beta}\right)
$$

where

$$
c(\sigma)=\min [1,1-2 \sigma], \quad b(\sigma)=\max [1,1-2 \sigma]
$$

or, equivalently

$$
\begin{array}{ll}
c(\sigma)=1, & b(\sigma)=1-2 \sigma(\dot{\sigma}<0) \\
c(\sigma)=1-2 \sigma, & b(\sigma)=1\left(0 \leqq \sigma<\frac{1}{2}\right) .
\end{array}
$$

Remarks analogous to those at the end of Section 2 can be made in this case also. Finally we note that corresponding results for generalized plane stress follow on formally replacing $\sigma$ by $\sigma /(1+\sigma)$ in the above $(\mu$ being unaltered).

\section{REFERENCES}

[1] C. O. Horgan, On Saint-Venant's Principle in Plane Anisotropic Elasticity, (to appear in Jnl. of Elasticity).

[2] J. K. Knowles and E. Sternberg, On Saint-Venant's Principle and the Torsion of Solids of Revolution, Archive for Rational Mechanics and Analysis, 22 (1966) 100-120.

[3] R. A. Toupin, Saint-Venant's Principle, Archive for Rational Mechanics and Analysis, 18 (1965) 83-96.

[4] J. J. Roseman, A Pointwise Estimate for the Stress in a Cylinder and its Application to Saint-Venant's Principle, Archive for Rational Mechanics and Analysis, 21 (1966) 23-48.

[5] I. S. Sokolnikoff, Mathematical Theory of Elasticity, Second Edition, McGraw-Hill, New York (1956).

[6] R. Bellman, Introduction to Matrix Analysis, McGraw-Hill, New York (1960).

[7] M. Marcus and H. Minc, A Survey of Matrix Theory and Matrix Inequalities, Allyn and Bacon, Boston (1964).

[8] R. J. Knops and L. E. Payne, Uniqueness Theorems in Linear Elasticity, Springer Tracts in Natural Philosophy, Volume 19. Springer-Verlag, Berlin (1971). 